\title{
Antworten auf die Kommentare von Greiner und Burda (Heft 2/2019)
}

\author{
Markus Erismann \\ Psychotherapie-Wissenschaft 10 (2) 81-83 2020 \\ www.psychotherapie-wissenschaft.info \\ CC BY-NC-ND \\ https://doi.org/10.30820/1664-9583-2020-2-81
}

\begin{abstract}
Zusammenfassung: Im vorliegenden Artikel setze ich mich mit den wissenschaftstheoretischen Kommentaren von Greiner (2019) und Burda (2019) auseinander. Greiner unterscheidet mit Bezug auf Wallner «Wissen» und «Erkenntnis». Dies entspricht meiner Auffassung von Reflexion auf die psychotherapeutische Praxis. Durch diese Reflexion wird «Erkenntnis» gewonnen. Hinsichtlich Burdas Konzept der Selbst-Differenz, das mehr auf Auflösung statt auf Bildung von Identität bedacht ist, stellt sich die Frage, wie dieses Konzept auf die psychotherapeutische Praxis anwendbar ist.
\end{abstract}

Schlüsselwörter: «Wissen» und «Erkenntnis», Selbst-Differenz, Identität, Reflexion, Gespräch

Im folgenden Artikel gebe ich Antworten zu Kurt Greiners Kommentar: «Akademische Psychotherapie, Psychotherapiewissenschaft und Experimentalhermeneutische Laborforschung» und Gerhard Burdas Kommentar: "Psychotherapie und Wissenschaft: eine Nabelschau?", die beide in Heft 2, 2019 der Psychotherapie-Wissenschaft erschienen sind.

Für Greiner (2019) ist die Reflexion zunächst ein "Funktionsprinzip», das in der psychotherapeutischen Praxis der "methodischen Schaffung von Wissen" dient. Mit Bezug auf Wallner unterscheidet er davon die «methodische Gewinnung von Erkenntnis» (ebd., S. 21). Dem entspricht Greiners Unterscheidung zwischen «Wissen in technisch-funktionaler Hinsicht (1. Konstitutionskriterium)» und "Erkenntnis in kritisch-reflexiver Hinsicht (2. Konstitutionskriterium)». Während das 1. Konstitutionskriterium einer Vielzahl von Wissensformen zukommt, zeichnet sich Akademische Wissenschaft darüber hinaus durch Erkenntnisgenerierung (2. Konstitutionskriterium) aus. Psychotherapie bedarf daher, um sich als Wissenschaft zu konstituieren, der «systematischen Produktion vertiefter Einsichten in die komplexen Strukturen ihrer professionellen Aktivitäten», wodurch sie "schliesslich erst differenzierte Erkenntnisse hinsichtlich impliziter Voraussetzungen und Bedingungen ihrer methodischen Wissenschaftsakte gewinnt»(ebd.). Diese Erkenntnisproduktion geschieht mittels wissenschaftshermeneutischer Reflexion auf das eigene professionelle Denken und Handeln.

Gemäss Greiner spielt die Reflexion für die Psychotherapie durch deren innerwissenschaftliche «Vielschichtigkeits- und Fremdartigkeitsstruktur» eine ausgezeichnete Rolle: «Faktisch liegt in keinem anderen wissenschaftlichen Fach eine dermassen hohe fachimmanente Reflexions-Provokation strukturbedingt vor, wie in der Psychotherapie» (ebd.). Ihre besondere Strukturiertheit und die damit verbundene Reflexions-Provokation bilden daher ein Abgrenzungskriterium und ein Qualitätsmerkmal der Psychotherapie.
Greiners prägnante Charakterisierung der Psychotherapie ist ganz im Sinne meiner Ausführungen. Wenn ich am Anfang meines ersten Artikels "Wissenschaftstheoretische Überlegungen zur Psychotherapiewissenschaft» mein Verständnis von Methodizität formuliere -

\footnotetext{
«Unter Methodizität verstehe ich die methodologische Reflektiertheit einer Wissenschaft, d.h. die Reflexion einer Wissenschaft auf die eigenen Grundlagen, ihre Grundbegriffe und Leitdifferenzen, die Art und Weise der Erkenntnisgewinnung, der Theoriebildung, der Strukturierung und Darstellung gewonnener Erkenntnisse sowie der Entwicklung von Anwendungsverfahren. Methodizität kann als Abgrenzungskriterium gegenüber anderen Arten von Wissen wie etwa dem Alltagswissen oder dem mythischen Wissen dienen» (Erismann, 2016, S. 7) -,
}

dann entspricht diese Auffassung m. E. genau dem von Greiner genannten 2. Konstitutionskriterium. Seiner kritischen Behauptung am Schluss seines Kommentars, ich bezöge mich bloss auf das 1 . Konstitutionskriterium (technisch-funktionales Wissen), möchte ich daher widersprechen. Im Gegenteil ist es so, dass mich gerade die Einsicht in die Notwendigkeit einer kritischen Reflexion auf die Psychotherapie und jede ihrer einzelnen Schulen zum Verfassen meiner beiden Artikel motiviert hat (zweiter Artikel: «Der Wissenschaftsbegriff der Psychotherapiewissenschaft»; Erismann, 2019).

Die scheinbare Divergenz verschwindet dadurch, dass deutlich zwischen der Reflexion als Funktionsprinzip der psychotherapeutischen Praxis (technisch-funktionale Wissensgenerierung) und der wissenschaftshermeneutischen Reflexion auf die Grundlagen der Psychotherapie (kritisch-reflexive Erkenntnisgenerierung) unterschieden wird. Die besondere Strukturiertheit der Psychotherapie und die mit ihr verbundene Reflexions-Provokation evozieren aber auch die Frage nach dem Zusammenhang der 
beiden Reflexionsformen: Inwiefern muss die Reflexion in der psychotherapeutischen Praxis mit einer Reflexion auf die wissenschaftlichen Grundlagen der entsprechenden psychotherapeutischen Schule einhergehen; wie muss diese zweifache Reflexion strukturiert sein, um sowohl Wissen als auch Erkenntnis zu generieren?

In dem von Greiner angeführten Verfahren der Experimentellen Trans-Kontextualisation sehe ich eine geeignete Möglichkeit, Grundelemente unterschiedlicher psychotherapeutischer Ansätze sichtbar zu machen, sie zu vergleichen und kritisch zu beurteilen. Die kritische Beurteilung hängt dann aber ihrerseits vom Standpunkt des/der die Konfrontation durchführenden Psychotherapiewissenschaftlers bzw. -wissenschaftlerin ab. Dieser Standpunkt wäre seinerseits einer kritischen Prüfung zu unterziehen.

Dazu eine Frage zum dialogexperimentellen Durchführungsbeispiel: Als Resultat der dialogischen Konfrontation zwischen Strukturaler Psychoanalyse und Transaktionsanalyse ergibt sich, dass aufgrund der unterschiedlichen Position des «anderen Subjekts» (hinter/ anstelle) der struktural-psychoanalytischen Intervention eine erfolgreiche transaktionsanalytische Therapie, die den «integrierten Erwachsenen-Ich-Zustand» herstellt, vorauszugehen habe. Dieses Resultat scheint mir durch die Perspektive der Transaktionsanalyse, in deren Kontext das struktural-psychoanalytische Transponat zu integrieren versucht wird, bestimmt zu sein. Was wäre aus der Perspektive der Strukturalen Psychoanalyse auf das Resultat der dialogischen Konfrontation zu antworten? Wird nicht durch die Festlegung des Herkunfts- und Verfremdungskontextes die eine Perspektive der anderen von vornherein bevorzugt?

Noch eine Bemerkung zum Verhältnis zwischen Psychotherapie und Psychotherapiewissenschaft: Da ich weder Psychotherapeut noch Psychotherapiewissenschaftler bin, stellt sich mir die Frage, inwieweit ich mich überhaupt in die Angelegenheiten der Psychotherapie einzumischen habe. Dazu fehlt mir eigentlich das nötige psychotherapeutische und psychotherapiewissenschaftliche Fachwissen. Aus dieser externen Perspektive kann es bestenfalls darum gehen, wissenschaftsphilosophische Anregungen zu einer Diskussion zu geben. Aufgrund meines distanzierten Standpunktes haben sich zudem terminologische Unklarheiten ergeben. Bislang war ich nämlich der Ansicht, der Verwissenschaftlichungsprozess der Psychotherapie habe die Anerkennung der Psychotherapiewissenschaft als eigenständige Wissenschaft zum Ziel. Gemäss Greiner handelt es sich aber darum, dass die Psychotherapie mithilfe der Psychotherapiewissenschaft zur Akademischen Psychotherapie akademisiert wird. Vermutlich geht es aber um beides: sowohl um die Akademisierung der Psychotherapie als auch um die Etablierung der Psychotherapiewissenschaft als eigenständige Wissenschaft.

Damit komme ich zu Burdas (2019) Kritik. Er formuliert seine Bedenken gegenüber meiner Erwartung an PsychotherapeutInnen, über ein ausgeprägtes selbstreflexives Bewusstsein hinsichtlich ihres therapeutischen Denkens und Handelns zu verfügen. Zudem zeigt er, wie diese angenommene Selbstreflexivität hinsichtlich einer wissenschaftstheoretischen Reflexion zu einem Zirkel führt. Dieser Zirkel ist gemäss Burda dadurch zu vermeiden, dass eine externe Perspektive, ein Blick von aussen, eingenommen wird und eine klare Trennung zwischen Psychotherapie und Psychotherapiewissenschaft vorgenommen wird. Beidem stimme ich zu.

Im Folgenden möchte ich begründen, weshalb ich dennoch an meiner Erwartung eines hohen Masses an Selbstreflexivität der Psychotherapie festhalte. Der erste Grund liegt in der ausgezeichneten Rolle, die die Reflexion in der Psychotherapie spielt. Greiner hat dies treffend als Reflexions-Provokation beschrieben. Bereits die Wahl einer bestimmten psychotherapeutischen Schule, sofern sie nicht blindlings erfolgt, verlangt ein bestimmtes Mass an (Selbst-)Reflexion auf die verschiedenen angebotenen psychotherapeutischen Ansätze und deren Grundlagen. Der zweite Grund liegt darin, dass die Psychotherapiewissenschaft nur dann als Mittlerin zwischen Wissenschaftstheorie und psychotherapeutischen Schulen fungieren kann, wenn die jeweilige Schule bzw. die einzelnen PsychotherapeutInnen offen genug sind, die Erkenntnisse der Psychotherapiewissenschaft aufzunehmen, wofür die Fähigkeit, sich infrage stellen zu lassen, Voraussetzung ist. Von dieser Fähigkeit hängt es ab, wie sich das Gespräch zwischen PsychotherapeutInnen und PsychotherapiewissenschaftlerInnen gestaltet.

Durch die Unterscheidung zwischen Wissens- und Erkenntnisgenerierung wird eine externe Perspektive, der Blick von aussen, konstituiert: die Psychotherapiewissenschaft, die sich dieser Unterscheidung von Wallner bedient, blickt von aussen auf die Psychotherapie und produziert durch diese Reflexion Erkenntnis. Nun können sich m. E. auch PsychotherapeutInnen von ihrer Tätigkeit distanzieren, aus der psychotherapeutischen Situation heraustreten und aus einer kritischen Distanz auf ihre psychotherapeutische Tätigkeit und deren Grundlagen reflektieren. Sie nehmen damit zumindest zeitweilig den Reflexionsstandpunkt der Psychotherapiewissenschaft ein und können auf die Hintergrundtheorie, das Menschenbild und die Grundauffassungen der Schule ihrer Wahl kritisch reflektieren. Dieser Reflexionsstandpunkt bedarf seinerseits nicht unbedingt einer philosophischen Theorie, wie sie der Kritische Realismus von Wallner darstellt. Dessen Unterscheidung zwischen Wissens- und Erkenntnisgenerierung, deren sich die Psychotherapiewissenschaft bedient, stellt sicher ein geeignetes Instrument dar, die kritisch-reflexive Position, den psychotherapiewissenschaftlichen Blick von aussen, zu festigen. Damit wird aber der Reflexionsstandpunkt an eine philosophische Theorie gebunden, die ihrerseits einer kritischen Prüfung bedarf. Weder die Einnahme eines (selbst-)kritischen Reflexionsstandpunktes, zu der PsychotherapeutInnen fähig sind oder sein sollten, noch die psychotherapiewissenschaftliche Reflexion auf die psychotherapeutische Theorie und Praxis bedürfen m. E. eines Bezugs zu einem bestimmten philosophischen Hintergrund.

Ich würde daher eher für eine grösstmögliche Unabhängigkeit der kritischen psychotherapeutischen und psycho- 
therapiewissenschaftlichen (Selbst-)Reflexion plädieren, die sich nicht an einen bestimmten philosophischen Hintergrund bindet. Dies entspricht ungefähr meiner eigenen Situation: Ich bin als Nicht-Psychotherapeut und NichtPsychotherapiewissenschaftler weder an eine bestimmte psychotherapeutische Schule noch an eine bestimmte philosophische Theorie gebunden. Meine angeblichen «Gewährsmänner», von denen der eine nebenbei bemerkt eine Frau ist (Elisabeth Wagner), sind bloss vage Orientierungspunkte für einen Reflexionsgang, der ohne feste philosophische Position eher einem work in progress entspricht.

Noch eine Bemerkung zum Konzept der Selbst-Differenz, das ich in (wissenschafts-)philosophischer Hinsicht interessant finde. Die Selbst-Differenz ist gemäss Burda ein allgemeines Prinzip, das jede Form von Identität betrifft. Jeder Wissensform ist eine inhärente Selbst-Differenz eigen. Dies zeigt Burda (2019) in einem informativen Abschnitt am Beispiel der Geschichte der Wissenschaftstheorie. Wissenschaft im Allgemeinen ist selbst-different, das heisst, sie bildet nach innen und aussen ein «komplexes Verbindungs- und Trennungsverhältnis» (ebd., S. 36). Dies gilt auch für die Psychotherapie und die Psychotherapiewissenschaft sowie ihr Verhältnis zueinander. Hinsichtlich der psychotherapeutischen Praxis, in der es unter anderem um die Rekonstruktion und Sicherung versehrter personaler Identität als Dimension eines intakten Selbstempfindens (Stern, 2007) bzw. eines Ich-Bewusstseins (Scharfetter, 1999) geht, frage ich mich, inwiefern dieses Konzept der Selbst-Differenz, das eher auf Auflösung statt auf Bildung von Identität bedacht ist, für die Psychotherapie als Heilverfahren angewandt werden kann.

Zum Schluss möchte ich drei Fragen stellen, die vielleicht für eine psychotherapiewissenschaftliche Forschung von Interesse sein könnten und für deren Beantwortung die Experimentalhermeneutische Laborforschung einen geeigneten Ansatz bietet:

1. Die Wahl einer bestimmten psychotherapeutischen Schule beinhaltet die Wahl einer bestimmten Perspektive, an der festzuhalten es für die PsychotherapeutInnen im Laufe ihrer Biografie eine gewisse Notwendigkeit gibt und die kaum mehr einen Perspektivenwechsel zulässt, das heisst die Einnahme der Perspektive anderer Schulen. Für ein Gespräch zwischen verschiedenen Schulen scheint mir aber die Fähigkeit, sich der anderen Perspektive öffnen zu können, eine Voraussetzung zu sein. Wie lässt sich die Gebundenheit der PsychotherapeutInnen an die eigene Perspektive überwinden?

2. Inwiefern bedarf die Fähigkeit zum Perspektivenwechsel des Vermögens, sich selbst und seine eigene (psychotherapeutische oder psychotherapiewissenschaftliche) Position und Tätigkeit infrage zu stellen bzw. stellen zu lassen? Dies setzt seinerseits angesichts der Beschränktheit der eigenen Perspektive Demut voraus.

3. Es kann die allgemeine Frage gestellt werden, wie Reflexion zu konzipieren und ein Gespräch zu strukturieren ist, um Wissen und Erkenntnis generieren zu können. Wie ist das Verhältnis zwischen Reflexion und Gespräch zu formulieren?

\section{Literatur}

Burda, G. (2019). Psychotherapie und Wissenschaft: eine Nabelschau? Psychotherapie-Wissenschaft, 9(2), 31-40.

Erismann, M. (2016). Wissenschaftstheoretische Überlegungen zur Psychotherapiewissenschaft. Psychotherapie-Wissenschaft, 6(1), 6-16.

Erismann, M. (2019). Der Wissenschaftsbegriff der Psychotherapiewissenschaft. Psychotherapie-Wissenschaft, 9(2), 13-17.

Greiner, K. (2019). Akademische Psychotherapie, Psychotherapiewissenschaft und Experimentalhermeneutische Laborforschung. Psychotherapie-Wissenschaft, 9(2), 20-28.

Scharfetter, C. (1999). Schizophrene Menschen. Weinheim: PVU.

Stern, D. (2007). Die Lebenserfahrung des Säuglings. Stuttgart: KlettCotta.

\section{Replies to the comments of Greiner and Burda}

Abstract: In this article I deal with the epistemological comments of Greiner (2019) and Burda (2019). Greiner differentiates between «Wissen» and «Erkenntnis» with reference to Wallner. This corresponds to my view of reflection on psychotherapeutic practice. «Erkenntnis» is gained by means of this reflection. With regard to Burda's concept of self-differentiation, which is more concerned with dissolution than with the formation of identity, the question arises, how this concept can be applied to psychotherapeutic practice.

Key words: «Wissen» and «Erkenntnis», self-differentiation, identity, reflection, dialogue

\section{Risposte alle osservazioni di Greiner e Burda}

Riassunto: Nel presente articolo vengono analizzate le osservazioni e i commenti teorico-scientifici esposti da Greiner (2019) e Burda (2019). Il primo autore, in riferimento a Wallner, pone l'accento sulla differenza tra «sapere» e «conoscenza». Ciò corrisponde alla mia posizione riguardante la riflessione sulla prassi psicoterapeutica. Mediante tale riflessione la «conoscenza» viene ampliata. In relazione al concetto del secondo autore, riguardante la differenziazione del sé, più in linea con una dissoluzione identitaria che con una sua formazione, sorge la domanda di come tale concetto possa essere impiegato nella prassi terapeutica.

Parole chiave: «sapere» e "conoscenza», differenziazione del sé, identità, riflessione, colloquio

\section{Der Autor}

Markus Erismann, Dr. phil., studierte von 1983 bis 1989 Philosophie und Germanistik an der Universität Zürich und promovierte 1995/96 am Philosophischen Seminar der Universität Zürich. Seine Arbeitsschwerpunkte sind Erkenntnistheorie und Methodologie.

\section{Kontakt}

Dr. Markus Erismann

Säntisstrasse 2

8008 Zürich

E-Mail: markus.erismann@bluewin.ch 